\title{
Activation of adrenergic receptor $\beta 2$ promotes tumor progression and epithelial mesenchymal transition in tongue squamous cell carcinoma
}

\author{
HAICHAO LIU ${ }^{1,2}$, CHENG WANG ${ }^{1,2}$, NAN XIE $^{2,3}$, ZEHANG ZHUANG ${ }^{1,2}$, XIQIANG LIU $^{1,2}$, \\ JINSONG HOU ${ }^{2,3}$ and HONGZHANG HUANG ${ }^{1,2}$
}

\begin{abstract}
${ }^{1}$ Department of Oral and Maxillofacial Surgery, Guanghua School of Stomatology, Hospital of Stomatology;
${ }^{2}$ Guangdong Provincial Key Laboratory of Stomatology; ${ }^{3}$ Department of Oral Pathology, Guanghua School of Stomatology, Hospital of Stomatology, Sun Yat-Sen University, Guangzhou, Guangdong 510055, P.R. China
\end{abstract}

Received January 26, 2016; Accepted October 31, 2017

DOI: $10.3892 /$ ijmm.2017.3248

\begin{abstract}
Tongue squamous cell carcinoma (TSCC) is more aggressive than other cancers in the head and neck region because of its potential for metastasis. Recently, $\beta 2$-adrenergic receptor $(\beta 2-\mathrm{AR})$ has been reported to be a potential promoter in various types of solid cancer. However, the role of $\beta 2-A R$ and its effect on TSCC is not well documented. Histological staining, western blot analysis, migration and invasion assay were used. In this study, the expression of $\beta 2$-AR was increased in TSCC tissue compared with adjacent non-cancerous epithelium. Further analysis demonstrated that increased expression of $\beta 2-A R$ was correlated with differentiation, lymph node metastasis and reduced overall survival rate in patients with TSCC. In vitro studies confirmed that activation of $\beta 2-\mathrm{AR}$ can promote epithelial mesenchymal transition in TSCC by initiating an interleukin-6/Stat3/Snail1 pathway. These results suggest that $\beta 2$-AR has an oncogenic role in TSCC and may be a potential therapeutic target in TSCC.
\end{abstract}

\section{Introduction}

Tongue squamous cell carcinoma (TSCC) is the most common malignancy in the oral cavity. TSCC is more aggressive than other cancers of the oral cavity because of the propensity for rapid local invasion and spread. Unfortunately, the mortality rate of TSCC has not improved significantly and the 5-year survival rate has remained at $<50 \%$ in the past 30 years

Correspondence to: Dr Hongzhang Huang, Department of Oral and Maxillofacial Surgery, Guanghua School of Stomatology, Hospital of Stomatology, Sun Yat-Sen University, 74 Zhongshan Second Road, Guangzhou, Guangdong 510055, P.R. China E-mail: drhuang52@163.com

Key words: $\beta 2$-adrenergic receptor, tongue squamous cell carcinoma, epithelial mesenchymal transition, interleukin-6 despite increasing advances in therapeutic modalities. The major cause of TSCC-associated mortalities is local/regional relapse and metastasis. Previous studies demonstrated that epithelial mesenchymal transition (EMT) has a pivotal role in cancer invasion and metastasis $(1,2)$. EMT is characterized by loss of cell polarity and adhesion, enhancement of cell motility and acquisition of a mesenchymal phenotype. It has been confirmed that reduced E-cadherin expression is a hallmark of EMT. The loss of E-cadherin has been linked with enhanced cell invasion and metastasis in oral SCC (3-5). Our previous studies demonstrated that E-cadherin expression was decreased in the budding tumor cells located ahead of invasive tumor front in TSCC, and expression was positively correlated with lymph node metastasis (6). Further studies confirmed that Snail2, histone-lysine N-methyltransferase EZH2 and microRNA-138 (7-9) regulate E-cadherin expression at the transcriptional and post-transcriptional levels. However, the molecular mechanisms underlining EMT process are still not fully understood in TSCC.

$\beta 2$-adrenergic receptor ( $\beta 2$-AR) is a seven transmembrane receptor (also known as $\mathrm{G}$ protein-coupled receptor) required for the physiological response to adrenaline and noradrenaline. Notably, several studies indicated that the $\beta 2$-AR pathway has an important role in solid cancer initiation and progression, including in breast cancer, malignant melanoma, prostate cancer and gastric cancer (10-13). More recent studies demonstrated that activation of $\beta 2-\mathrm{AR}$ signaling was involved in EMT, and contributed to invasion and metastasis in colorectal adenocarcinoma and gastric cancer (14-16). However, the expression of $\beta 2-\mathrm{AR}$ and its effect on TSCC progression have not well been documented. In this study, deregulation of $\beta 2-\mathrm{AR}$ in TSCC was examined to investigate its roles in EMT and TSCC progression.

\section{Materials and methods}

Reagents and antibodies. Anti- $\beta 2-\mathrm{AR}$ (cat. no. ab182136) was purchased from Abcam (Cambridge, UK), anti-interleukin-6 (IL-6; cat. no. 12153), anti-E-cadherin (cat. no. 9101s), anti-vimentin (cat. no. 9102s), anti-Snail (cat. no. 3879), 
anti-phospho-signal transducer and activator of transcription 3 (Stat3; cat.no.12640) and anti-GAPDH(cat.no.2118s)antibodies were purchased from Cell Signaling Technology, Inc. (Danvers, MA, USA). Human IL-6 antibody (cat. no. AF-206-NA) were purchased from R\&D Systems, Inc. (Minneapolis, MN, USA). Non-selective $\beta$-AR blocker, propranolol hydrochloride (cat. no. 318-98-9), non-specific $\beta$-AR agonist, isoproterenol hydrochloride (cat. no. 51-30-9), the $\beta 1$-AR antagonist, metoprolol tartrate (cat. no. 56392-17-7) and the $\beta 2-\mathrm{AR}$ antagonist ICI-118,551 hydrochloride (cat. no. 72795-19-8), were purchased from Sigma-Aldrich (Merck KGaA, Darmstadt, Germany). Anti-rabbit IgG, HRP-linked secondary antibody (cat.no. 7074s) was purchased from Cell Signaling Technology.

Patients and tissue samples. All tissue samples were collected between January 2007 and December 2009, obtained from the First Affiliated Hospital, Sun Yat-Sen University (Guangzhou, China). Patients with TSCC $(n=75)$ were enrolled in this study. Adjacent non-cancerous tissue samples $(n=20)$ and TSCC specimens $(n=75)$ were used for immunohistochemical assessment. All tissue samples used in this study were used following the guidelines set by the Institution Review Board of Sun Yat-Sen University. All patients received radical surgery and none received any form of adjuvant therapy prior to surgery. The tumor extent was classified based on the TNM system by Union for International Cancer Control, and the tumor grade was classified following the World Health Organization classification of histological differentiation (6). Survival was calculated according to the date of surgery and the date of the last follow-up (or death). Informed consent was signed by all patients for the use of their tissue and clinical data in clinical analysis and research studies prior to treatment.

Immunohistochemistry and staining evaluation. Immunohistochemical staining was performed according to standard protocols (7). Samples were fixed in $4 \%$ paraformaldehyde for $24 \mathrm{~h}$ at room temperature and embedded in paraffin. Briefly, $4 \mu \mathrm{m}$ thick tissue sections were dewaxed and hydrated routinely. Following antigen retrieval, tissue sections were incubated with primary antibodies at $4^{\circ} \mathrm{C}$ overnight. Samples were washed for five times with phosphate-buffered saline, then incubated with secondary antibody (1:200) at room temperature for $30 \mathrm{~min}$. Tissues were treated with 3,3'-diaminobenzidine (1:200) for $1 \mathrm{~min}$ at room temperature and counterstained with hematoxylin for $10 \mathrm{sec}$ at room temperature. After dehydration, slides were observed and analyzed using an Axioskop 40 (Carl Zeiss AG, Oberkochen, Germany) microscope. Five fields were randomly selected for each specimen under a light microscope at a magnification of $x 400$, and three experienced observers analyzed the images. The scoring criteria were according to a standard protocol described previously (7). A final score $>4$ was classified as high $\beta 2$-AR expression and a score $\leq 4$ was classified as low $\beta 2$-AR expression.

Cell cultures and treatments. Cal27 and SCC15 TSCC cell lines were obtained from American Type Culture Collection (Manassas, VA, USA). Cells were cultured in Dulbecco's modified Eagle's medium-F12 (Gibco: Thermo Fisher Scientific, Inc., Waltham, MA, USA) supplemented with $10 \%$ fetal bovine serum (HyClone; GE Healthcare Life Sciences, Logan, UT, USA), $1 \%$ penicillin and streptomycin. All cells were incubated at $37^{\circ} \mathrm{C}$ in a humidified atmosphere containing $5 \% \mathrm{CO}_{2}$ with the culture media changed every 2 days. Cultured cells were treated at 70 to $90 \%$ confluence and were serum-starved for $8 \mathrm{~h}$ before treatment. Briefly, cells were treated for $48 \mathrm{~h}$ according the groups as follows: i) Culture medium (control); ii) $1 \mu \mathrm{M}$ propranolol (Prop); iii) $1 \mu \mathrm{M}$ propranolol $+1 \mu \mathrm{M}$ isoproterenol (Prop + ISO); iv) $1 \mu \mathrm{M}$ metoprolol $+1 \mu \mathrm{M}$ isoproterenol (Meto + ISO); v) $1 \mu \mathrm{M}$ ICI-118,551 $+1 \mu \mathrm{M}$ isoproterenol (ICI + ISO); and vi) $1 \mu \mathrm{M}$ ISO. Propranolol, metoprolol and ICI-118,551 were added in the culture media $30 \mathrm{~min}$ before adding ISO.

IL-6/Stat3 signaling analysis. Cancer cells were cultured with medium containing $500 \mathrm{ng} / \mathrm{ml}$ IL-6 antibody (R\&D Systems, Inc.) to neutralize and block IL-6 signaling in TSCC cells.

Immunofluorescence and laser scanning confocal microscopy. Samples were blocked with goat serum (Boster Biological Technology, Wuhan, China) and incubated with the primary antibody [E-cadherin (1:250), vimentin (1:250)] diluted in 5\% bovine serum albumin (BSA; Boster Biological Technology) at $4^{\circ} \mathrm{C}$ overnight. Secondary antibody conjugated to fluorescein isothiocyanate was used to bind to the primary antibody (1:200) diluted in PBS at $37^{\circ} \mathrm{C}$ for $30 \mathrm{~min}$. Nuclei were stained with DAPI (1:250; Sigma-Aldrich; Merck KGaA) diluted in $\mathrm{PBS}$ at $37^{\circ} \mathrm{C}$ for $5 \mathrm{~min}$. Samples were placed on glass coverslip. Cells were observed using a confocal laser scanning microscope (Zeiss LSM 510 Meta; Carl Zeiss AG).

Migration and invasion assay. Cell migration assay was evaluated by using wound healing and Transwell membrane chambers ( $8 \mu \mathrm{m}$ pore size; Corning Inc., New York, NY, USA). The wound healing assay was performed as previously described (16). Briefly, the cells were seeded in 12-well plates and treated with ISO at $90 \%$ confluence and normal culture medium was added to the control cells. For Transwell membrane chambers, cells were seeded into the upper chambers and cultured with or without ISO for $36 \mathrm{~h}$. For invasion assay, top chambers coated with Matrigel (BD Biosciences, Franklin Lakes, NJ, USA) at a concentration of $1.37 \mathrm{mg} / \mathrm{ml}$. Upper chambers were fixed in $4 \%$ paraformaldehyde at room temperature for $30 \mathrm{~min}$, stained using $0.1 \%$ crystal violet at room temperature for $5 \mathrm{~min}$, then counted using a Axioskop 40 (Carl Zeiss AG) microscope.

Western blot analysis. Cells were lysed on ice in $120 \mu 1$ radioimmunoprecipitation lysis buffer containing phosphatase inhibitors (Sigma-Aldrich; Merck KGaA) for $30 \mathrm{~min}$. Total proteins ( $30 \mu \mathrm{g}$; determined by bicinchoninic acid assay) were loaded and separated by $10 \%$ SDS-PAGE, and then transferred into nitrocellulose membrane (EMD Millipore, Billerica, MA, USA). Membranes were blocked in 5\% evaporated skimmed milk at room temperature for $1 \mathrm{~h}$ and then incubated with primary antibody at $4^{\circ} \mathrm{C}$ overnight. Primary antibodies $(1: 1,000)$ were diluted in $5 \%$ evaporated skimmed milk at $4^{\circ} \mathrm{C}$ overnight. Then incubated the membrane with horseradish peroxidase-conjugated secondary antibody (1:2,000; diluted in 5\% evaporated skimmed milk at room temperature for $1 \mathrm{~h}$; Cell Signaling Technology, Inc.) and observed using chemiluminescence (EMD Millipore).

Statistical analysis. Data was presented as mean \pm SD. All experiments were performed at least three times and all 

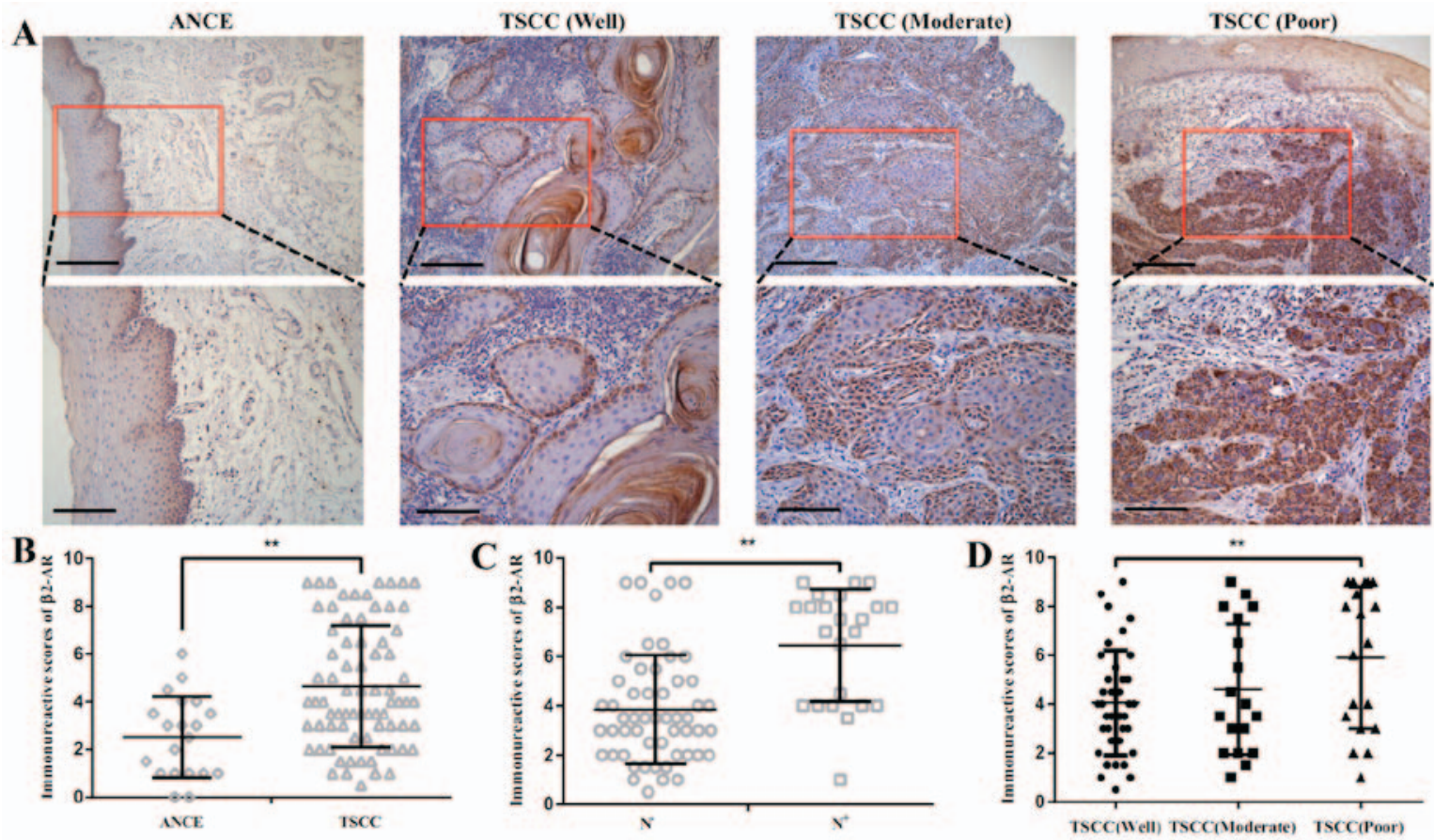

Figure 1. Expression of $\beta 2-\mathrm{AR}$ in TSCC samples. (A) Expression of $\beta 2$-AR in ANCE and TSCC samples (upper images scale bar, $50 \mu \mathrm{m}$; lower images scale bar, $20 \mu \mathrm{m}$ ). (B) Semi-quantitative analysis revealed that $\beta 2$-AR is overexpressed in TSCC compared with ANCE. (C) Expression of $\beta 2-A R$ was enhanced in TSCC with lymph node metastasis when compared with TSCC samples without lymph node metastasis. (D) Expression of $\beta 2$-AR was increased in TSCC tissue with poor differentiation than with well differentiation. Scale bar, $50 \mu \mathrm{m}$. Error bars represent the standard deviation; ${ }^{* *} \mathrm{P}<0.01$. $\beta 2$-AR, $\beta 2$-adrenergic receptor; TSCC, tongue squamous cell carcinoma; ANCE, adjacent non-cancerous epithelium.

statistical analyses were performed using SPSS 13.0 software for Windows (SPSS, Inc., Chicago, IL, USA). The association between $\beta 2$-AR expression and the clinical pathological parameters was determined by $\chi^{2}$ test. The survival analysis was performed by the Kaplan-Meier method. Student's t-test was used for statistical analysis. $\mathrm{P}<0.05$ was considered to indicate a statistically significant difference. A Cox regression was used to analyze the association between the survival time and the clinicopathological variables.

\section{Results}

Expression of $\beta 2-A R$ in TSCC samples. The expression of p- $\beta 2-A R$ was detected by immunohistochemistry in 75 cases with TSCC. The $\beta 2-A R$ was detected in at low levels in adjacent non-cancerous epithelium (ANCE). In primary tumor samples, predominant cytoplasm and membrane staining of $\beta 2-\mathrm{AR}$ was detected in cancer cells (Fig. 1A). The semi-quantitative analysis revealed that $\beta 2-A R$ was overexpressed in TSCC compared with ANCE (Fig. 1B). Enhanced expression of $\beta 2$-AR was also observed in TSCC with lymph node metastasis compared with TSCC samples without lymph node metastasis (Fig. 1C). $\beta 2$-AR expression was increased SCC tissue with poor differentiation compared with well differentiated TSCC tissue (Fig. 1D). Among 75 cases examined in the study, $36(48 \%)$ cases exhibited high $\beta 2-\mathrm{AR}$ staining and $40(52 \%)$ cases displayed low $\beta 2$-AR expression.

Association of $\beta 2-A R$ with clinicopathological features in TSCC.Associations were tested between $\beta 2$-AR expression and clinical and pathological features in this TSCC cohort (Table I). $\chi^{2}$ analysis demonstrated that $\beta 2$-AR expression was associated
Table I. Association between $\beta 2-\mathrm{AR}$ expression and clinicopathological features in patients with tongue squamous cell carcinoma.

$\beta 2-A R$ expression

Clinicopathologic No. of

feature cases High (\%) Low (\%) P-value

Gender

Male

38

19

19

0.725

Female

37

17

20

Age (years)

$\geq 55$

40

21

19

0.404

$<55$

35

15

20

Differentiation

$\begin{array}{llll}\text { Well } & 40 & 13 & 27 \\ \text { Moderate+poor } & 35 & 23 & 12\end{array}$

T stage

$\mathrm{T}_{1-2}$

68

$\mathrm{T}_{3-4}$

7

34

2

34

$$
5
$$

$\mathrm{N}$ stage

$$
\mathrm{N}^{-}
$$

$\mathrm{N}^{+}$

52

20

32

$16 \quad 7$

Clinical stage

$\begin{array}{llll}\text { I-II } & 48 & 20 & 28 \\ \text { III-IV } & 27 & 16 & 11\end{array}$

0.143

0.004

$\beta 2$-AR, $\beta 2$-adrenergic receptor. 
Table II. Cox proportional analysis of variables affecting survival in patients with tongue squamous cell carcinoma.

\begin{tabular}{|c|c|c|c|c|c|}
\hline \multirow{2}{*}{$\overline{\text { Variable }}$} & \multirow[b]{2}{*}{ Comparison } & \multicolumn{2}{|c|}{ Univariate analysis } & \multicolumn{2}{|c|}{ Multivariate analysis } \\
\hline & & HR $(95 \% \mathrm{CI})$ & $\mathrm{P}$-value & $\mathrm{HR}(95 \% \mathrm{CI})$ & P-value \\
\hline Gender & Male vs. female & $1.059(0.475-2.359)$ & 0.889 & & \\
\hline Age (years) & $\geq 55$ vs. $<55$ & $0.996(0.442-2.246)$ & 0.992 & & \\
\hline Differentiation & Well vs. moderate or poor & $0.712(0.319-1.588)$ & 0.406 & & \\
\hline pT stage & $\mathrm{T}_{1-2}$ vs. $\mathrm{T}_{3-4}$ & $2.392(0.893-6.409)$ & 0.083 & & \\
\hline pN stage & $\mathrm{N}^{0}$ vs. $\mathrm{N}^{+}$ & $3.070(1.372-6.871)$ & 0.006 & $0.878(0.168-4.596)$ & 0.877 \\
\hline pTNM stage & I-II vs. III-IV & $3.039(1.346-6.860)$ & 0.007 & $2.823(0.582-13.696)$ & 0.198 \\
\hline$\beta 2-\mathrm{AR}$ expression & High vs. low & $3.220(1.396-7.428)$ & 0.006 & $2.780(1.090-7.086)$ & 0.032 \\
\hline
\end{tabular}

pTNM stage, pathological tumor node metastasis stage; $\beta 2$-AR, $\beta 2$-adrenergic receptor; HR, hazard ratio; CI, confidence interval.

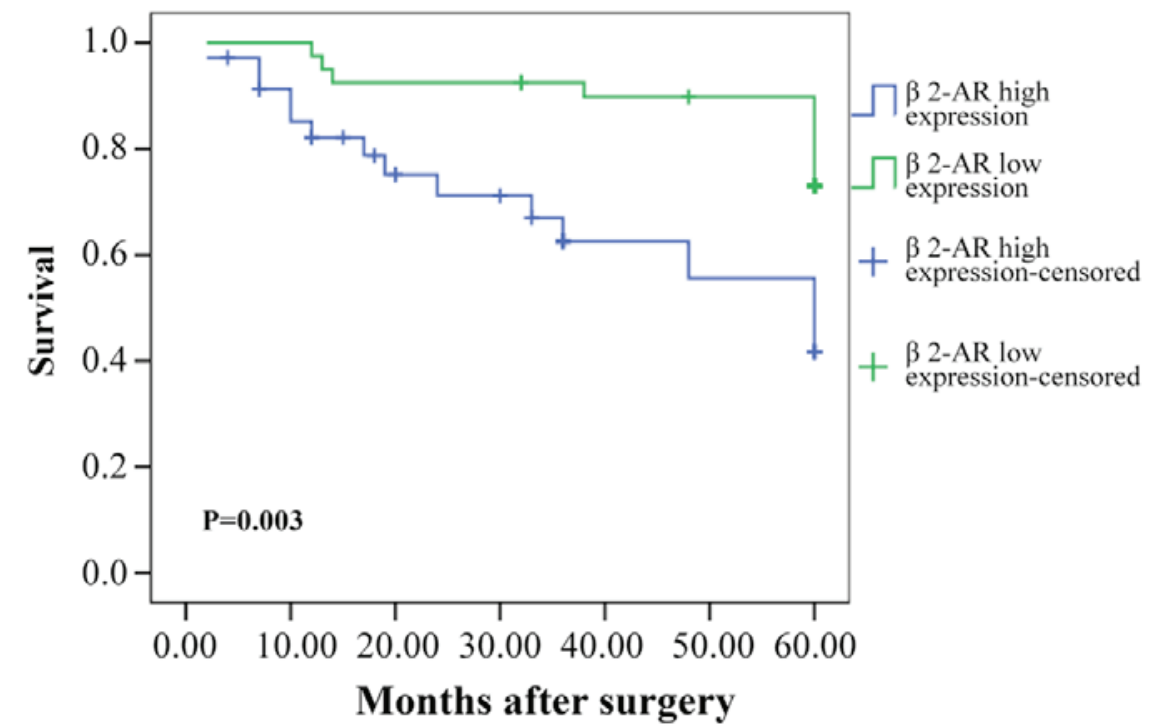

Figure 2. Kaplan-Meier analysis. Kaplan-Meier analysis demonstrate a statistically significant difference in 5-year survival rate between high $\beta 2$-AR expression group and low $\beta 2$-AR expression group. P-value was determined using the log-rank test. $\beta 2$-AR, $\beta 2$-adrenergic receptor.

with tumor differentiation $(\mathrm{P}=0.004)$ and $\mathrm{N}$ stage $(\mathrm{P}=0.013)$. There were no associations between $\beta 2$-AR expression and age, gender, $\mathrm{T}$ stage and clinical stage.

Prognostic value of $\beta 2-A R$ for patients with TSCC. Kaplan-Meier analysis demonstrated a statistically significant difference in 5-year survival rate between patients with high $\beta 2$-AR expression and low $\beta 2$-AR expression $(\mathrm{P}=0.003$; Fig. 2). As demonstrated in Table II, univariate analysis indicated that tumor size, grade, pathological T stage, lymph node metastasis, clinical stage, and $\beta 2$-AR were all significant prognostic factors for survival of patients with TSCC. Multivariate analysis showed that lymph node metastasis, clinical stage, and $\beta 2-A R$ were all independent prognostic factors for patient survival.

Effect of activation of $\beta 2-A R$ on EMT in TSCC cells. TSCC cell lines Cal27 and SCC15 were treated with ISO ( $\beta$-AR agonist) to activate $\beta 2$-AR signaling. The cell morphology was dramatically changed from an epithelial phenotype to mesenchymal phenotype with decreased E-cadherin expression and increased vimentin expression (Fig. 3A and B). A wound healing assay indicated that these changes were accompanied by enhanced cell motility (Fig. 4A and B). Similar results were also observed in Transwell assay coated with or without Matrigel (Fig. 5A and B). Western blot analyses further confirmed that E-cadherin expression was downregulated and vimentin expression was upregulated by treatment with ISO (Fig. 5C and D). By contrast, western blot analysis indicated that ISO-mediated activation of $\beta 2-A R(p-\beta 2-A R)$, increased expression of vimentin and Snail1, and reduced E-cadherin expression were inhibited by co-treatment with ICI-118,551, but not metoprolol (Fig. 6A and B). The expression of IL-6, Stat3, p-Stat3 and Snail1 were also examined in TSCC cells treated by ISO with or without ICI-118,551. Activation of $\beta 2-A R$ promoted the expression of IL- 6 , p-Stat 3 and Snail1, while blockade of $\beta 2$-AR signaling with ICI-118,551 inhibited $\beta 2$-AR-mediated activation of IL-6/Stat3/Snail 

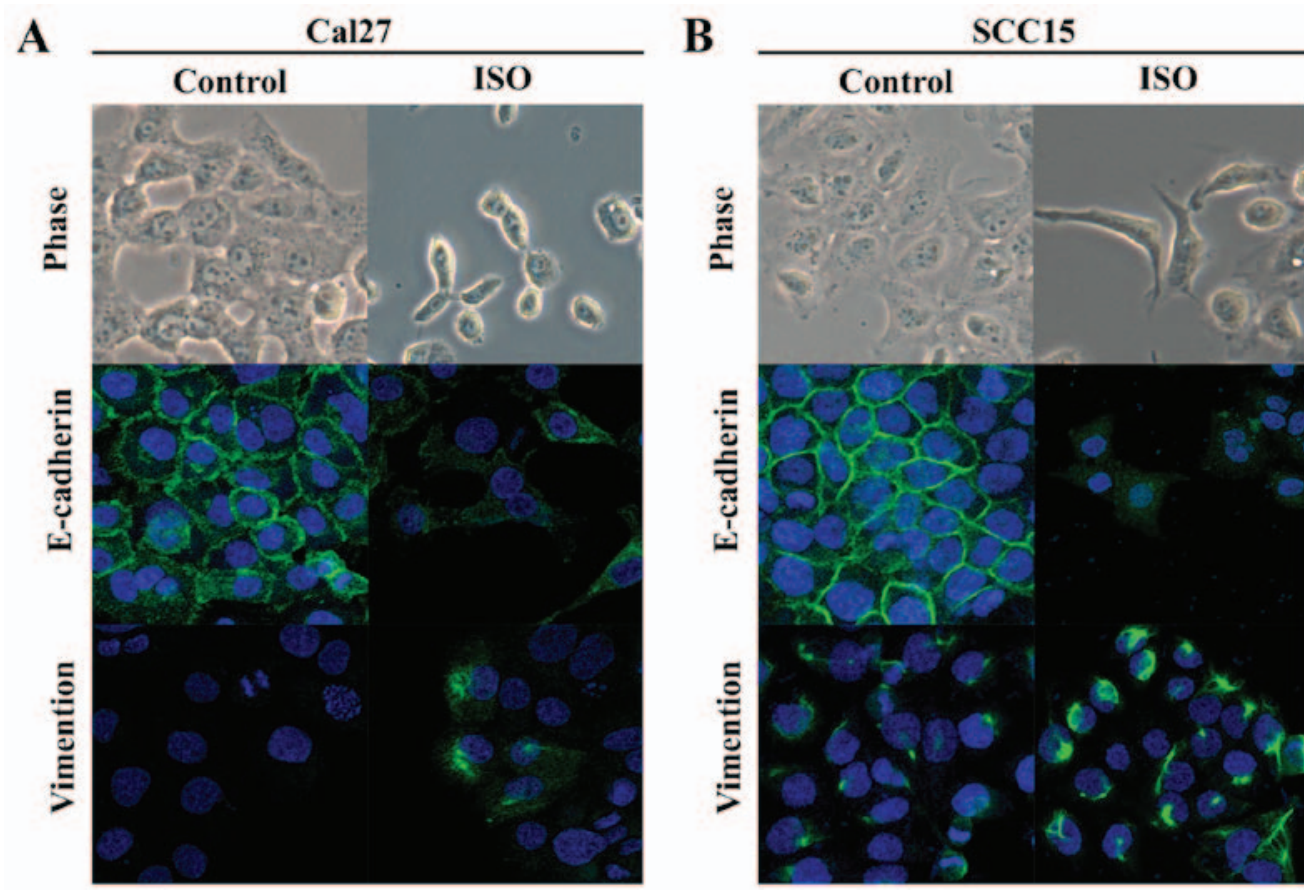

Figure 3. Light microscope and laser scanning confocal microscopy observations indicate that ISO induced epithelial mesenchymal transition morphological alterations in Cal27 and SCC15 cell lines. (A) Cal27 and (B) SCC15 cell lines, cell morphology was dramatically changed from epithelial phenotype to mesenchymal phenotype. E-cadherin expression was decreased and vimentin expression was increased. ISO, isoproterenol.

A
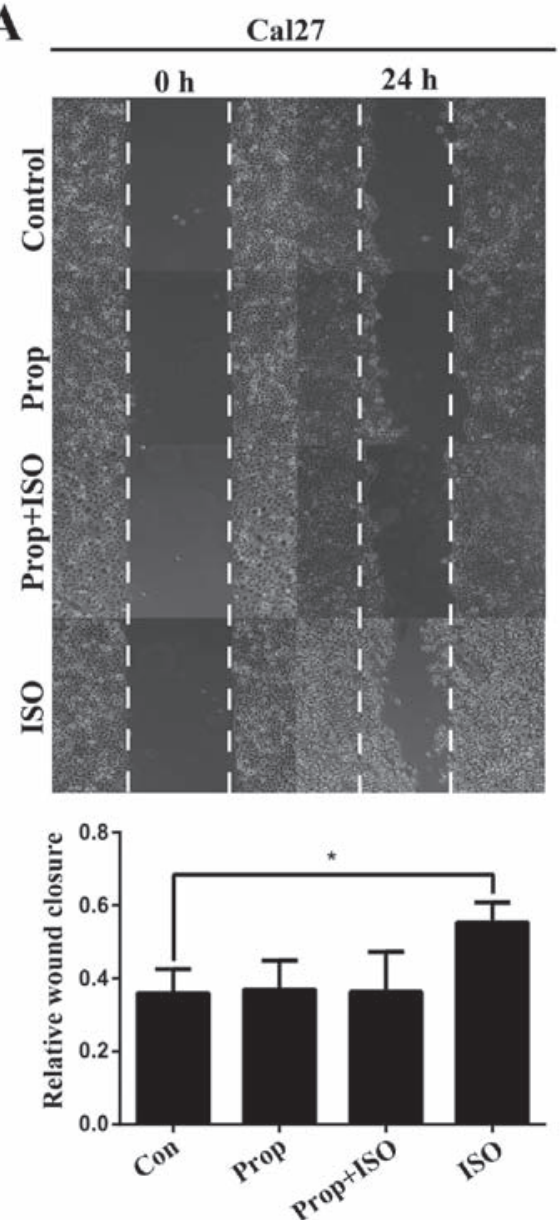

B
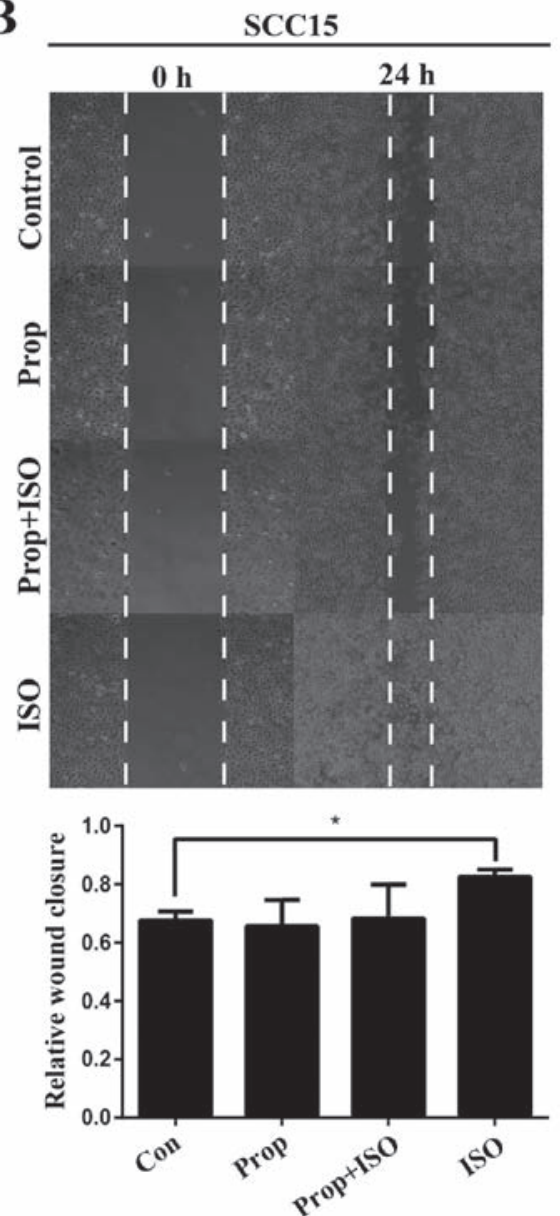

Figure 4. Wound healing assay. (A) In Cal27 cell lines and (B) SCC15 cell lines, the ability of wound healing was increased in ISO-treated groups compared with controls and the ability was similar in Prop + ISO group and control groups. Error bars represent the standard deviation; * $<<0.05$. ISO, isoproterenol; Prop, propranolol. 
A

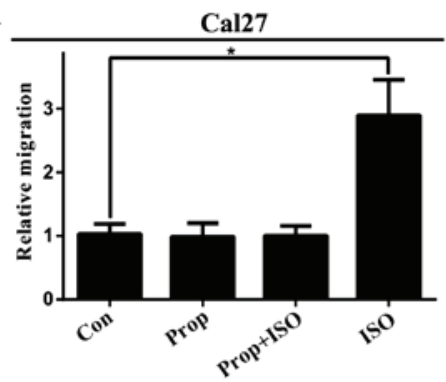

Cal27

B
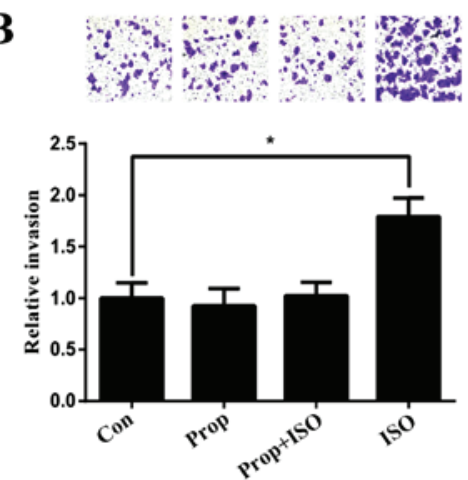

C

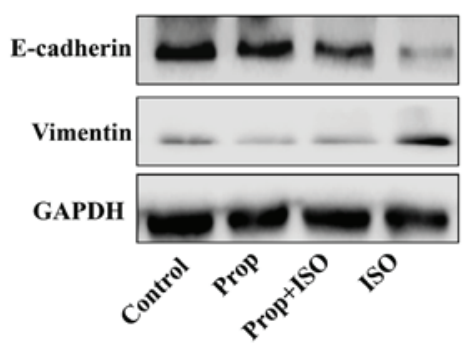

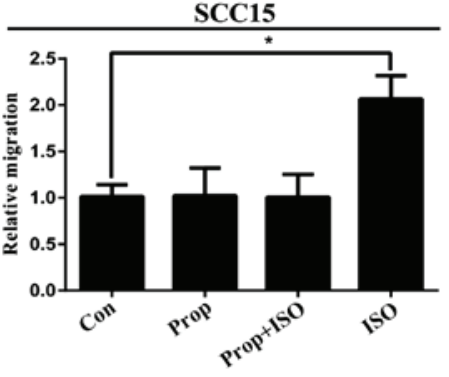

SCC15
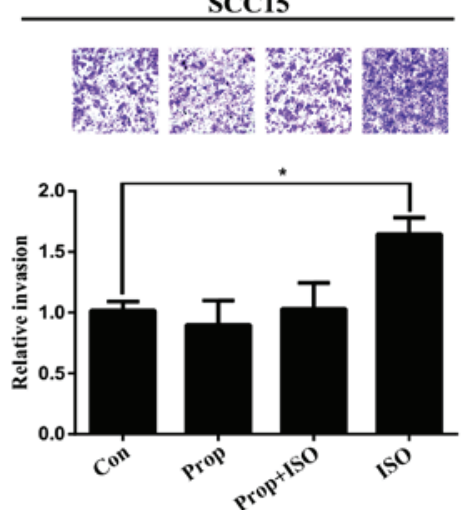

D

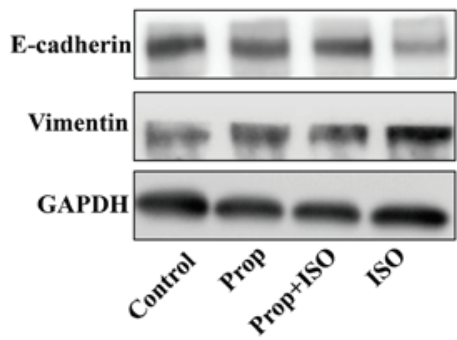

Figure 5. Prop attenuates ISO-stimulated Cal27 and SCC15 cell migration and invasion by inhibiting ISO-induced epithelial mesenchymal transition. Representative, (A) ISO increased cells migration and invasion and attenuated by Prop in Cal27 and (B) SCC15. Western blot analysis indicated that, (C) in Ca127 and (D) SCC15 cells, E-cadherin expression was downregulated and vimentin expression was upregulated in ISO exposed groups than controls. Error bars represent the standard deviation; ${ }^{*} \mathrm{P}<0.05$. Prop, propranolol; ISO, isoproterenol.

A

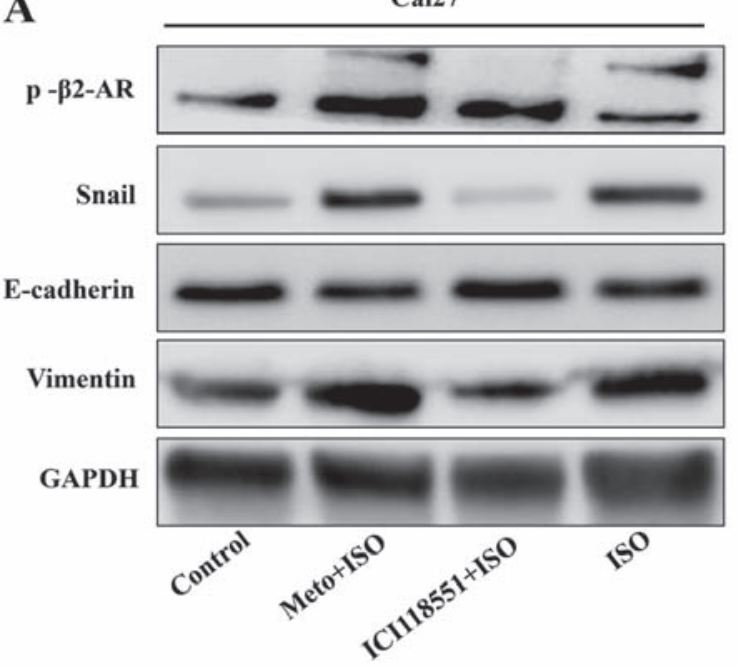

B
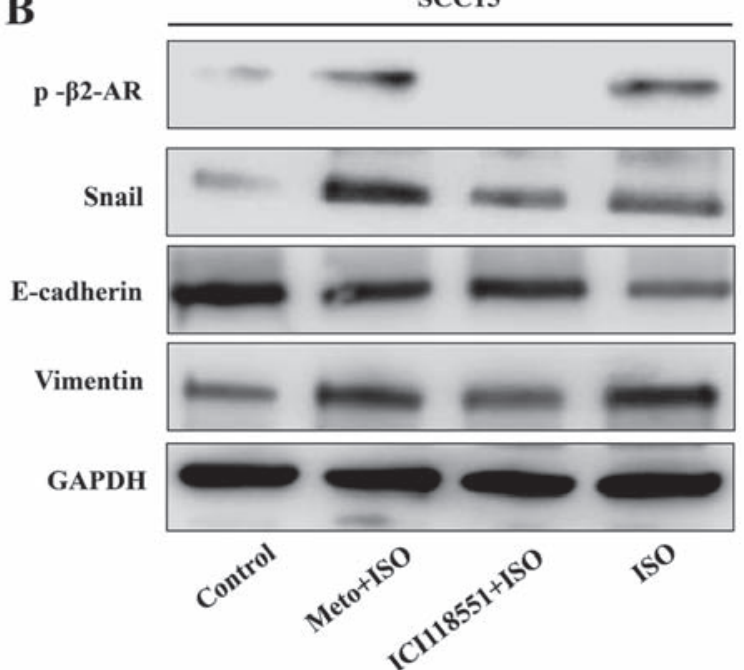

Figure 6. Western blot analysis indicates that ISO induced epithelial mesenchymal transition through mediating activation of $\beta 2$-AR. (A) In Cal27 and (B) SCC15 cell lines, p- $\beta 2-A R$, vimentin and Snail1 were increased in ISO-treated groups, while E-cadherin expression were decreased. Following treatment with ICI-118,551 but not Meto, ISO induced the expression of vimentin and Snail1, and E-cadherin was attenuated. p-, phospho; $\beta 2$-AR, $\beta 2$-adrenergic receptor Meto, metoprolol; ISO, isoproterenol. 
A

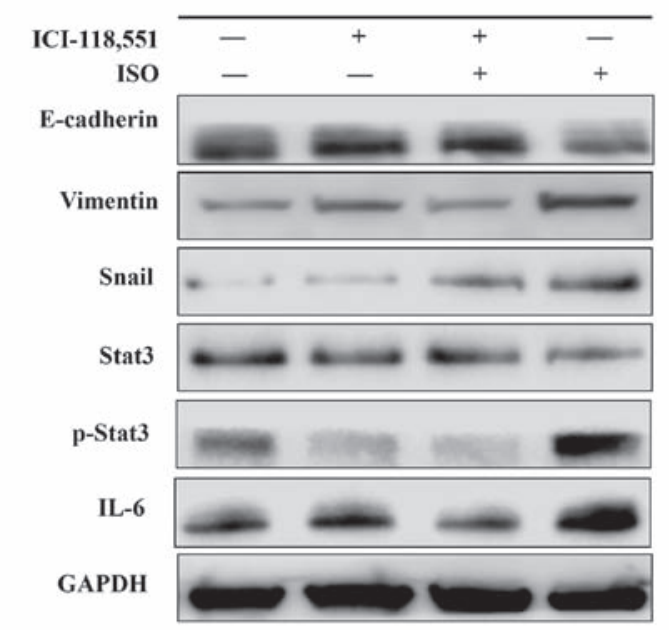

SCC15

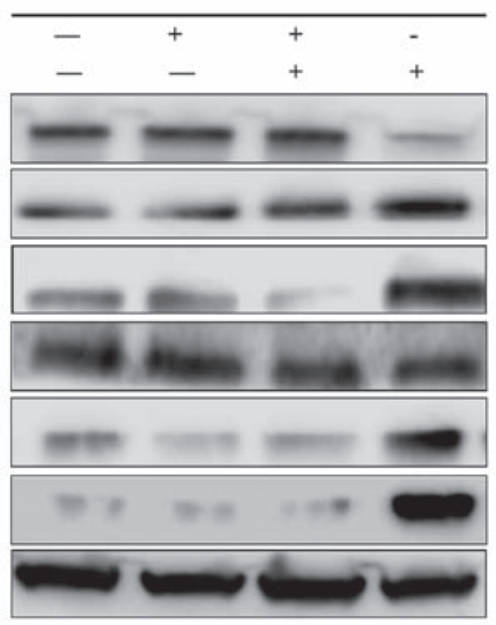

B

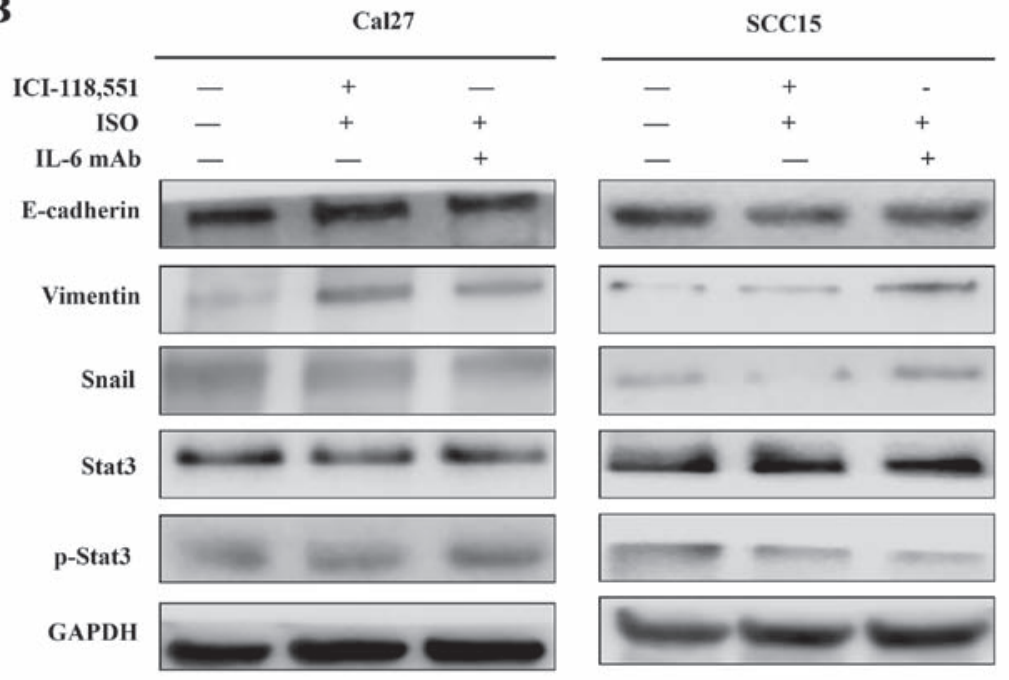

Figure 7. Activation of $\beta 2$-AR promoted epithelial mesenchymal transition in Cal27 and SCC15 cells through initiating IL-6/Stat3/Snail1 pathway. (A) Expression of IL-6, Stat3, p-Stat3 and Snail1 in Cal27 and SCC15 cells treated by ISO with or without ICI-118,551. (B) Expression of IL-6, Stat3, p-Stat3 and Snail1 in the presence of IL-6 mAb, ISO and ICI-118,551. ISO, isoproterenol; $\beta 2$-AR, $\beta 2$-adrenergic receptor; IL-6, interleukin-6; mAb, monoclonal antibody; Stat3, signal transducer and activator of transcription 3; p-, phospho.

pathway (Fig. 7A). When cells were treated with anti-IL-6 monoclonal antibody (m Ab), ISO-induced EMT was partially reversed by blocking IL-6/Stat3/Snail pathway (Fig. 7B) (24).

\section{Discussion}

Accumulating data demonstrate that EMT has an important role in malignant cancer progression $(17,18)$. Cancer cells can acquire the ability to migrate and invade during EMT, which is orchestrated by a complex molecular network. Recently, stress has been reported to be correlated with cancer growth, drug resistance, recurrence, progression and poor prognosis of patients (12). Stress activates the sympathetic nervous system and results in increased production of catecholamines, such as norepinephrine and epinephrine (12). The activation of $\beta 2$-AR signaling was indicated to be responsible for stress-mediated cancer progression $(19,20)$. The oncogenic role of $\beta 2$-AR has been suggested in several cancers type, including oral cancer $(21,22)$. However, the deregulation of $\beta 2$-AR in TSCC and its role in invasion and metastasis of TSCC has not been fully investigated.
In the present study, the expression of $\beta 2$-AR was examined in TSCC tissue samples, and it was confirmed that $\beta 2$-AR expression was enhanced in TSCC tissue compared with non-cancerous adjacent epithelium. This observation indicates that deregulation of $\beta 2$-AR signaling may contribute to TSCC tumorigenesis. Similar results were previously observed in other studies (22). Further analysis demonstrated that increased expression of $\beta 2$-AR was positively associated with lymph node metastasis, clinical stage and reduced overall survival. Furthermore, overexpression of $\beta 2-\mathrm{AR}$ was an independent poor prognostic factor in patients with TSCC. These findings indicated that activation of $\beta 2$-AR signaling has a critical role in the invasion and metastasis cascade in TSCC. Notably, Bravo-Calderón et al (21) reported that strong expression of $\beta 2$-AR was a favorable prognostic factor for patients with OSCC.

To confirm the observations above and explore the functional relevance of $\beta 2$-AR in invasion and metastasis of TSCC, TSCC cells were treated with ISO ( $\beta$-AR agonist) and/or propranolol (non-selective $\beta$-AR antagonist) to activate or block $\beta$-AR signaling pathway; a switch of TSCC cells from epithelial 
to mesenchymal phenotype was observed in cancer cells, which suggested that EMT was induced when cancer cells were treated with ISO. The switch was also accompanied by decreased expression of E-cadherin, increased expression of vimentin and enhanced cell migration and invasion. These changes were also abolished by treatment with propranolol. To further confirm that activation of $\beta 2$-AR was responsible for the ISO-induced EMT, metoprolol (selective $\beta 1-A R$ antagonist) and ICI-118,551 (selective $\beta 2$-AR antagonist) were used to inhibit $\beta 1-A R$ and $\beta 2-A R$ signaling, respectively. Inhibition of $\beta 2$-AR reversed ISO-induced EMT. These findings confirmed that activation of $\beta 2-A R$ induced EMT to promote migration and invasion in TSCC cells. Similar results were also reported by Lu et al (15) and Shan et al (16). However, the molecular mechanisms of $\beta 2$-AR-mediated EMT in TSCC are not clear. Bernabé et al (23) reported that $\beta$-AR agonists, such as norepinephine, and ISO stimulation both increase IL-6 secretion in human oral SCC cells lines. As previously reported (24-26), the IL-6/Stat3 pathway has an important role in EMT. To further clarify the potential mechanism of $\beta 2-\mathrm{AR}$ induced EMT, IL-6/Stat3/Snail1 pathways were investigated in our study. In the current study, increased production of IL-6 was observed in Cal27 and SCC15 cells following ISO stimulation, which was also associated with activation of Stat 3 and increased Snail1 expression to initiate EMT. Notably, these molecular changes can be blocked by treatment with IL-6 mAb. These results suggested that activation of IL-6/Stat3/Snail pathway is involved in $\beta 2$-AR mediated EMT in oral SCC.

In conclusion, the results of the present study demonstrated that deregulation of $\beta 2$-AR signaling may be involved in TSCC progression. One of its major roles is promoting EMT to enhance cancer cell migration and invasion in TSCC progression. Furthermore, $\beta 2$-AR-mediated EMT was regulated in TSCC, at least in part, through activation of an IL-6/Stat3/Snail pathway. Further studies are necessary to investigate the crosstalk between $\beta 2$-AR and IL-6 signaling.

\section{Acknowledgements}

This study was support by National Natural Science Grant of China (nos. 81272949, 81172567 and 81372885), Guangdong Province Nature Science Foundation (no. 20130319c).

\section{References}

1. Shaul YD, Freinkman E, Comb WC, Cantor JR, Tam WL, Thiru P, Kim D, Kanarek N, Pacold ME, Chen WW, et al: Dihydropyrimidine accumulation is required for the epithelial-mesenchymal transition. Cell 158: 1094-1109, 2014.

2. Thiery JP, Acloque H, Huang RY and Nieto MA: Epithelial-mesenchymal transitions in development and disease. Cell 139: 871-890, 2009.

3. Jiang Y, Liao L, Shrestha C, Ji S, Chen Y, Peng J, Wang L, Liao E and Xie Z: Reduced expression of E-cadherin and p120-catenin and elevated expression of PLC- $\gamma 1$ and PIKE are associated with aggressiveness of oral squamous cell carcinoma. Int J Clin Exp Pathol 8: 9042-9051, 2015.

4. Rajwar YC, Jain N, Bhatia G, Sikka N, Garg B and Walia E: Expression and significance of cadherins and its subtypes in development and progression of oral cancers: a review. J Clin Diagn Res 9: ZE05-ZE07, 2015.

5. Soares MQ, Mendonça JA, Morais MO, Leles CR, Batista AC and Mendonça EF: E-cadherin, $\beta$-catenin, and $\alpha 2 \beta 1$ and $\alpha 3 \beta 1$ integrin expression in primary oral squamous cell carcinoma and its regional metastasis. Histol Histopathol 30: 1213-1222, 2015.

6. Wang $C$, Huang H, Huang Z, Wang A, Chen X, Huang L, Zhou X and Liu X: Tumor budding correlates with poor prognosis and epithelial-mesenchymal transition in tongue squamous cell carcinoma. J Oral Pathol Med 40: 545-551, 2011.
7. Wang C, Liu X, Huang H, Ma H, Cai W, Hou J, Huang L Dai Y, Yu T and Zhou X: Deregulation of Snai2 is associated with metastasis and poor prognosis in tongue squamous cell carcinoma. Int J Cancer 130: 2249-2258, 2012.

8. Wang C, Liu X, Chen Z, Huang H, Jin Y, Kolokythas A, Wang A, Dai Y, Wong DT and Zhou X: Polycomb group protein EZH2-mediated E-cadherin repression promotes metastasis of oral tongue squamous cell carcinoma. Mol Carcinog 52: 229-236, 2013

9. Liu X, Wang C, Chen Z, Jin Y, Wang Y, Kolokythas A, Dai Y and Zhou X: MicroRNA-138 suppresses epithelial-mesenchymal transition in squamous cell carcinoma cell lines. Biochem J 440: 23-31, 2011.

10. Liu D, Deng Q, Sun L, Wang T, Yang Z, Chen H, Guo L, Liu Y, Ma Y, Guo N, et al: A Her2-let-7- $\beta 2-A R$ circuit affects prognosis in patients with Her2-positive breast cancer. BMC Cancer 15: 832, 2015.

11. Yang EV, Kim SJ, Donovan EL, Chen M, Gross AC, Webster Marketon JI, Barsky SH and Glaser R: Norepinephrine upregulates VEGF, IL-8, and IL-6 expression in human melanoma tumor cell lines: implications for stress-related enhancement of tumor progression. Brain Behav Immun 23: 267-275, 2009.

12. Barbieri A, Bimonte S, Palma G, Luciano A, Rea D, Giudice A, Scognamiglio G, La Mantia E, Franco R, Perdonà S, et al: The stress hormone norepinephrine increases migration of prostate cancer cells in vitro and in vivo. Int J Oncol 47: 527-534, 2015.

13. Shi M, Yang Z, Hu M, Liu D, Hu Y, Qian L, Zhang W, Chen H, Guo L, Yu M, et al: Catecholamine-induced $\beta 2$-adrenergic receptor activation mediates desensitization of gastric cancer cells to trastuzumab by upregulating MUC4 expression. J Immunol 190: 5600-5608, 2013.

14. Zhang J, Deng YT, Liu J, Wang YQ, Yi TW, Huang BY, He SS, Zheng B and Jiang Y: Norepinephrine induced epithelial-mesenchymal transition in HT-29 and A549 cells in vitro. J Cancer Res Clin Oncol 142: 423-435, 2016.

15. Lu YJ, Geng ZJ, Sun XY, Li YH, Fu XB, Zhao XY and Wei B: Isoprenaline induces epithelial-mesenchymal transition in gastric cancer cells. Mol Cell Biochem 408: 1-13, 2015.

16. Shan T, Cui X, Li W, Lin W, Li Y, Chen X and Wu T: Novel regulatory program for norepinephrine-induced epithelial-mesenchymal transition in gastric adenocarcinoma cell lines. Cancer Sci 105: 847-856, 2014.

17. Ye X and Weinberg RA: Epithelial-mesenchymal plasticity: a central regulator of cancer progression. Trends Cell Biol 25: 675-686, 2015.

18. Ye X, Tam WL, Shibue T, Kaygusuz Y, Reinhardt F, Ng Eaton E and Weinberg RA: Distinct EMT programs control normal mammary stem cells and tumour-initiating cells. Nature 525: 256-260, 2015.

19. Qin JF, Jin FJ, Li N, Guan HT, Lan L, Ni H and Wang Y: Adrenergic receptor $\beta 2$ activation by stress promotes breast cancer progression through macrophages M2 polarization in tumor microenvironment. BMB Rep 48: 295-300, 2015.

20. Braadland PR, Ramberg H, Grytli HH and Taskén KA: $\beta$-Adrenergic receptor signaling in prostate cancer. Front Oncol 4: 375, 2015.

21. Bravo-Calderón DM, Oliveira DT, Marana AN, Nonogaki S, Carvalho AL and Kowalski LP: Prognostic significance of beta-2 adrenergic receptor in oral squamous cell carcinoma. Cancer Biomark 10: 51-59, 2011-2012.

22. Shang ZJ, Liu K and Liang DF: Expression of beta2-adrenergic receptor in oral squamous cell carcinoma. J Oral Pathol Med 38: 371-376, 2009

23. Bernabé DG, Tamae AC, Biasoli ÉR and Oliveira SH: Stress hormones increase cell proliferation and regulates interleukin-6 secretion in human oral squamous cell carcinoma cells. Brain Behav Immun 25: 574-583, 2011.

24. Yadav A, Kumar B, Datta J, Teknos TN and Kumar P: IL-6 promotes head and neck tumor metastasis by inducing epithelial-mesenchymal transition via the JAK-STAT3-SNAIL signaling pathway. Mol Cancer Res 9: 1658-1667, 2011.

25. Kim MS, Lee WS, Jeong J, Kim SJ and Jin W: Induction of metastatic potential by TrkB via activation of IL6/JAK2/STAT3 and PI3K/AKT signaling in breast cancer. Oncotarget 6: 40158-40171, 2015.

26. Liu H, Ren G, Wang T, Chen Y, Gong C, Bai Y, Wang B, Qi H, Shen J, Zhu L, et al: Aberrantly expressed Fra-1 by IL-6/STAT3 transactivation promotes colorectal cancer aggressiveness through epithelial-mesenchymal transition. Carcinogenesis 36 : 459-468, 2015.

This work is licensed under a Creative Commons Attribution-NonCommercial-NoDerivatives 4.0 International (CC BY-NC-ND 4.0) License. 